\title{
High D-dimer levels after stopping anticoagulants in pulmonary embolism with sleep apnoea
}

\author{
Angela García Suquia ${ }^{1}$, Alberto Alonso-Fernández ${ }^{2,3}$, Mónica de la Peña ${ }^{2,3}$, \\ David Romero ${ }^{4}$, Javier Piérola ${ }^{5}$, Miguel Carrera ${ }^{2,3}$, Antonia Barceló ${ }^{1,3}$, \\ Joan B. Soriano ${ }^{6}$, Meritxell Arque ${ }^{5}$, Carmen Fernández-Capitán ${ }^{7}$, \\ Alicia Lorenzo ${ }^{7}$ and Francisco García-Río 3,4
}

\begin{abstract}
Affiliations: ${ }^{1}$ Dept of Clinical Analysis, University Hospital Son Espases, Palma de Mallorca, Spain. ${ }^{2}$ Dept of Pneumology, University Hospital Son Espases, Palma de Mallorca, Spain. ${ }^{3}$ CIBER Enfermedades Respiratorias, Palma de Mallorca, Illes Balears, Spain. 'Dept of Pneumology, University Hospital La Paz, IdiPAZ, Madrid, Spain. ${ }^{5}$ IdISPa-FISIB, Hospital Universitari Son Espases, Palma de Mallorca, Spain. ${ }^{6}$ Instituto de Investigación Hospital Universitario de la Princesa (IISP), Universidad Autónoma de Madrid, Cátedra UAMLinde, Madrid, Spain. ${ }^{7}$ Dept of Internal Medicine, University Hospital La Paz, Madrid, Spain.
\end{abstract}

Correspondence: Alberto Alonso-Fernández, Servicio de Neumología. Hospital Universitario Son Espases, Carretera de Valldemossa 79, 07010 Palma de Mallorca, Spain. E-mail: alberto.alonsodassib.es

ABSTRACT Obstructive sleep apnoea is a risk factor for pulmonary embolism. Elevated D-dimer levels and other biomarkers are associated with recurrent pulmonary embolism. The objectives were to compare the frequency of elevated D-dimer levels $\left(>500 \mathrm{ng} \cdot \mathrm{mL}^{-1}\right)$ and further coagulation biomarkers after oral anticoagulation withdrawal in pulmonary embolism patients, with and without obstructive sleep apnoea, including two control groups without pulmonary embolism.

We performed home respiratory polygraphy. We also measured basic biochemical profile and haemogram, and coagulation biomarkers (D-dimer, prothrombin fragment $1+2$, thrombin-antithrombin complex, plasminogen activator inhibitor 1 , and soluble P-selectin).

$64(74.4 \%)$ of the pulmonary embolism cases and $41(46.11 \%)$ of the controls without pulmonary embolism had obstructive sleep apnoea. Plasmatic D-dimer was higher in PE patients with OSA than in those without obstructive sleep apnoea. D-dimer levels were significantly correlated with apnoeahypopnoea index, and nocturnal hypoxia. There were more patients with high D-dimer after stopping anticoagulants in those with pulmonary embolism and obstructive sleep apnoea compared with $\mathrm{PE}$ without obstructive sleep apnoea (35.4\% versus $19.0 \%, \mathrm{p}=0.003)$. Apnoea-hypopnoea index was independently associated with high D-dimer.

Pulmonary embolism patients with obstructive sleep apnoea had higher rates of elevated D-dimer levels after anticoagulation discontinuation for pulmonary embolism than in patients without obstructive sleep apnoea and, therefore, higher procoagulant state that might increase the risk of pulmonary embolism recurrence.

@ERSpublications

Pulmonary embolism patients with sleep apnoea are more likely to have elevated postanticoagulation plasma D-dimer http://ow.ly/NHoiu

This article has supplementary material available from erj.ersjournals.com

Received: Nov 042014 | Accepted after revision: May 092015 | First published online: July 232015

Support statement: This research was partially supported by grants from Direcció General d'Avaluació i Acreditació. Conselleria de Salut i Consum. Illes Balears 2009 (DGAVAL PI 040/09 (CAIB 09)), Neumomadrid 2009, SEPAR 2008 (820), SEPAR 2010 (820) and Ministerio de Educación y Ciencia (SAF2007-62270) and Ministerio de Economía y Competitividad (PI10/00495). Funding information for this article has been deposited with FundRef.

Conflict of interest: None declared.

Copyright @ERS 2015 


\section{Introduction}

There is increasing evidence from cross-sectional and longitudinal studies that obstructive sleep apnoea (OSA) is a risk factor for pulmonary embolism (PE) independent of potential confounders [1-5].

The annual incidence of PE is one to two cases per 1000 person-years, and it is strongly age dependent. $\mathrm{PE}$ and OSA share risk factors (advanced age, physical inactivity and obesity) [6, 7], its association represent a major public health burden, given its high prevalence and increased mortality rates $[7,8]$, which is likely to rise as the population ages and obesity rates increase in developing and emerging countries. Patients with a first episode of PE have a cumulative recurrence rate circa to $25 \%$ at 5 years [9]. Recurrent PE is associated with mortality rates of $4-9 \%$, and it is also a risk factor for chronic pulmonary hypertension. Anticoagulants are highly effective in decreasing the PE recurrence rate; however, it increases the risk of bleeding complications and, therefore, the optimal length of anticoagulation following PE remains unclear.

D-dimer is a fibrin degradation product that is used to evaluate patients with suspected PE. It has also reported a higher risk of $\mathrm{PE}$ recurrence in patients with unprovoked $\mathrm{PE}$ who had a high post-anticoagulation $\mathrm{D}$-dimer $\left(>500 \mathrm{ng} \cdot \mathrm{mL}^{-1}\right)$ than in patients with normal D-dimer levels [10]. A landmark randomised clinical trial found that patients with an abnormal D-dimer test who restarted anticoagulation had a significantly lower combined incidence of recurrent venous thromboembolism and bleeding than those who did not resume the treatment [11]. Thus, D-dimer has been proposed as a tool in guiding the length of therapy for PE.

The prognostic role of other biomarkers of haemostasis/fibrinolysis is less known. Elevated levels of prothrombin fragment $1+2(\mathrm{~F} 1+2)$ and soluble P-selectin (sP-selectin) have been explored as a risk factor for first and recurrent thromboembolic events, but studies have reported conflicting results [12-15].

Based on the aforementioned features and considering that OSA leads to a hypercoagulable state, we hypothesised that those patients discontinuing anticoagulation after a PE with OSA could have a greater risk of embolic recurrence, evidenced by the presence of a high level of $\mathrm{D}$-dimer, than non-apnoeic subjects. The main objective of the present study was to compare the frequency of elevated plasma levels $\left(>500 \mathrm{ng} \cdot \mathrm{mL}^{-1}\right)$ of D-dimer after oral anticoagulation withdrawal for a first PE episode in patients with and without OSA, including two control groups without PE, one with OSA and one with no sleep-breathing problems.

\section{Methods}

\section{Subjects, design and ethics}

We performed a prospective matched case-control study (University Hospital Son Espases, Palma de Mallorca and University Hospital La Paz, Madrid, Spain). Eligible cases were all patients with a previous (6-12 months) PE episode diagnosed by computed tomography (CT) pulmonary angiography that had completed at least 3 months with a vitamin $\mathrm{K}$ antagonist. They were included if they stopped anticoagulation for at least 1 month. Patients were excluded if they were unwilling or unable to participate in the study, estimated survival $<12$ months, and/or severe daytime hypoxaemia (arterial oxygen tension $<60 \mathrm{mmHg}$ ).

Controls were population-based volunteers. They were enrolled from the population area of the Primary Care Centres. They were never previously evaluated with a sleep study. We randomly selected a subject without previous diagnosis or clinical suspicion of thromboembolic disease, with similar in sex, age ( \pm 2 years), weight $( \pm 2 \mathrm{Kg})$ and height $( \pm 5 \mathrm{~cm})$ with regard to the two preceding PE patients included in the study and who had no exclusion criteria e.g. a very serious illness (terminal or serious physical/mental disability) that might prevent monitoring and participation in the study, inability to sign the informed consent and/or treatment with vitamin $\mathrm{K}$ antagonists, and those with communication or comprehension difficulties.

The study was approved by the Institutional Ethics Committee at the hospital and all subjects (cases and controls) gave their written informed consent.

\section{Measurements}

A validated portable recording sleep monitoring system (Stardust II; Respironics, Inc., Murrysville, PA, USA) was used to perform a sleep study in all patients and control subjects. Every patient or control was classified as having OSA when the obstructive component was dominant and apnoea-hypopnoea index (AHI) was $\geqslant 5 \cdot h^{-1}$ (see online supplementary material).

In all subjects anthropometric, clinical and sleep data were collected. We recorded, for all subjects, all the potential confounders which included: sex, age, body mass index (BMI), neck diameter, physical activity level, classic risk factors for pulmonary embolism (prolonged immobilisation major surgery, trauma, cancer, major medical diseases, hormone replacement, oral contraceptive therapy and cerebrovascular disease), blood pressure values, and forced expiratory volume in $1 \mathrm{~s}$ (FEV1) measured as \% predicted. Physical activity was measured with a self-administered questionnaire (International Physical Activity Questionnaire 
long form). Office blood pressure was measured by a random zero sphygmomanometer. More details and descriptions of each of the respiratory events are available in the online supplementary material.

In $\mathrm{PE}$ patients 1 month after oral anticoagulation withdrawal, a venous blood sample was collected. Quantitative determination of D-dimer levels was performed by inmunoturbidimetric assay (INNOVANCE D-Dimer of SIEMENS Healthcare, Marburg, Germany). D-dimer was considered high when $>500 \mathrm{ng} \cdot \mathrm{mL}^{-1}$. Plasma levels of soluble P-selectin (sP-selectin), thrombin-antithrombin complex (TAT), plasminogen activator inhibitor 1 (PAI-1), and F1+2, were determined by an enzyme-linked immunoassay (MAGPIX Multiplex Immunoassay, Merck Millipore, Dormstadt, Germany) according to manufacturer's instructions with Xponent software 4.1. Commercial kit R\&D Systems Quantikine (Minneapolis, MN, USA) was used to measure sP-selectin (Human soluble P-Selectin/CD62P Immunoassay) and PAI-1 (Human Serpin E1/PAI1) and for the quantitative determination of human prothrombin fragment F1+2 and TAT the commercial kit Enzygnost (SIEMENS Healthcare Diagnostics, Marburg, MN, Germany) was used. Measurements were always done in duplicate and the measured values were used for analysis. Intra-assay/inter-assay coefficient variation $(\mathrm{CV})$ for the techniques used were: sP-selectin $(5.2-8.8 \% \mathrm{CV})$; TAT (5-7.5\% CV); PAI-1 (6.7-7.3\% CV); F1+2 (4.5-7.8\% CV); D-dimer (4.2-5\% CV).

\section{Statistical analysis}

The normality of the distribution of variables was tested using the Kolmogorov-Smirnov test. Continuous variables were expressed as mean $\pm \mathrm{SD}$ or median (interquartile range), depending on their distribution. Categorical variables were reported as absolute numbers and percentages. Differences between study groups for qualitative and quantitative variables were analysed by the Chi-squared test and analysis of variance with Bonferroni post hoc test, respectively. The relationship between plasma levels of biomarkers and sleep parameters was determined using multiple linear regression analysis after normalisation of all variables by log transformation and adjustment for classic risk factors for PE. To examine associations between study groups and the study variables, odds ratios (OR) and 95\% confidence intervals (CI) in univariate and multivariate analyses were calculated by logistic regression. Statistical significance was assumed for $\mathrm{p}<0.05$. All analyses were performed using the Statistical Package for the Social Sciences, version 13.0 software (SPSS Inc., Chicago, IL, USA).
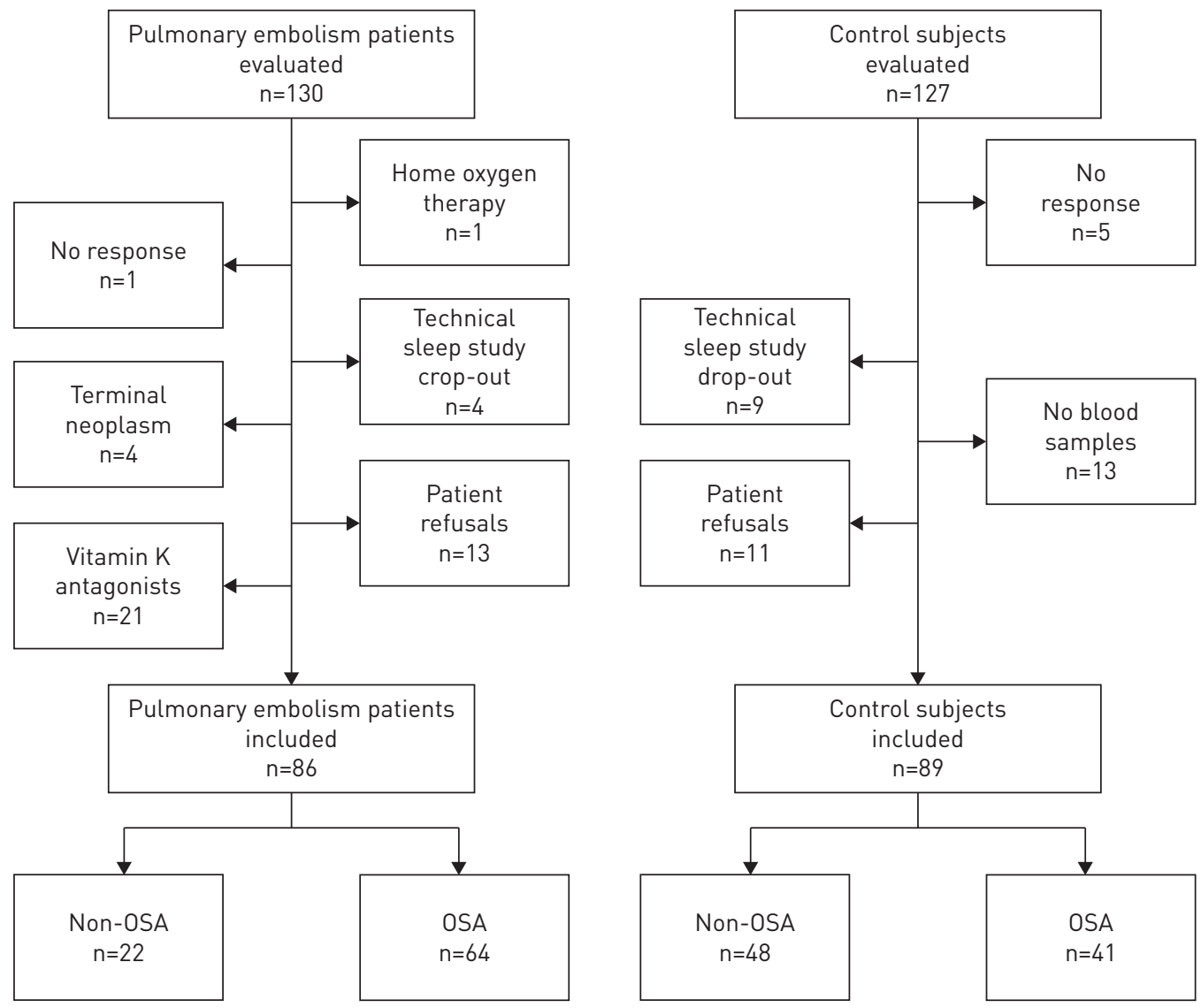

FIGURE 1 Flow diagram of all the study subjects. 
Results

From the 107 patients with PE and the 102 control subjects [1] we excluded 21 PE patients, who were on vitamin $\mathrm{K}$ antagonist, and 13 control subjects, who were without blood samples. All patients were under oral anticoagulation with acenocoumarol. Therefore, 86 patients with PE and 89 control subjects were finally included in the study (figure 1). There were no significant differences between patients with PE and control subjects in the anthropometric characteristics (age, sex, BMI, smoking, and alcohol intake).

\section{General characteristics}

Overall, 64 (74.4\%) PE patients and 41 (46.1\%) control subjects had OSA (apnoea-hypopnoea index $>5 \cdot \mathrm{h}^{-1}$ ) (figure 1 ). Subjects were classified in four study subgroups: 1) PE-OSA (PE patients with OSA), 2) PE non-OSA (PE patients without OSA), 3) C-OSA (control subjects with OSA) and 4) C non-OSA (control subjects without OSA). The four subgroups were homogenous in sex, BMI and daily physical activity level. OSA patients (PE-OSA and C-OSA) were older than non-OSA subjects (tables 1 and S1).

\section{Sleep study variables}

By definition AHI was significantly higher in OSA patients (PE-OSA and C-OSA) compared with non-OSA. In addition, OSA patients (PE-OSA and C-OSA) had worse nocturnal oxygen saturation indices than non-OSA subjects. PE-OSA also had higher desaturation index and lower mean night-time arterial oxygen saturation $\left(\mathrm{SaO}_{2}\right)$ than C-OSA $\left(22.9 \pm 17.2\right.$ event $\cdot \mathrm{h}^{-1}$ versus $15.7 \pm 14.0$ event $\cdot \mathrm{h}^{-1}$, and $92.6 \pm 2.4 \%$ versus $94.0 \pm 1.9 \%$, respectively; $\mathrm{p}<0.05$ ) (table 1 ). Lastly, no differences were found in daytime somnolence.

TABLE 1 Comparison of the demographic characteristics, sleep parameters, daily physical activity and plasma biomarkers between the study groups according to the presence of obstructive sleep apnoea (OSA)

\begin{tabular}{|c|c|c|c|c|c|}
\hline & \multicolumn{2}{|c|}{ PE patient group } & \multicolumn{2}{|c|}{ Control group } & p-value \\
\hline Subjects $n$ & 64 & 22 & 41 & 48 & \\
\hline Sex male & $43(67)$ & $9(41)$ & $31(66)$ & $28(51)$ & 0.066 \\
\hline Age years & $60 \pm 14^{\# \# \#, \text { ๆา }}$ & $50 \pm 16$ & $61 \pm 12^{\# \# \#, \text { กा }}$ & $49 \pm 14$ & 0.000 \\
\hline Body mass index $\mathrm{kg} \cdot \mathrm{m}^{-2}$ & $27.8 \pm 5.6^{\# \# \#}$ & $26.8 \pm 4.8$ & $27.5 \pm 3.9^{\#}$ & $25.7 \pm 3.9$ & 0.168 \\
\hline Epworth Sleepiness Scale & $7.1 \pm 3.9$ & $5.8 \pm 3.5$ & $6.0 \pm 3.9$ & $6.5 \pm 2.5$ & 0.359 \\
\hline Mean $\mathrm{SaO}_{2} \%$ & $92.6 \pm 2.4^{\# \# \#, \text { กाๆ१,+ }}$ & $94.9 \pm 1.2^{\#}$ & $94.0 \pm 1.9^{\# \# \#}$ & $95.5 \pm 1.4$ & 0.000 \\
\hline Desaturation index events: $\mathrm{h}^{-1}$ & $15.0(10.5-35.0)^{\# \# \#, 9191,+}$ & $2.7(2.2-4.0)$ & 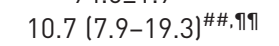 & $3.1(1.9-5.4)$ & 0.000 \\
\hline Minimum $\mathrm{SaO}_{2} \%$ & 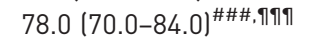 & $88.0(84.5-89.5)$ & 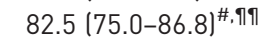 & $86(80.3-89.0)$ & 0.000 \\
\hline Physical activity level & & & & & 0.440 \\
\hline Low & 36.6 & 14.3 & 23.1 & 31.8 & \\
\hline Moderate & 41.5 & 71.4 & 51.3 & 40.9 & \\
\hline $\mathrm{HDL}$ cholesterol $\mathrm{mg} \cdot \mathrm{dL}^{-1}$ & $49 \pm 12^{\# \# \#,+}$ & $49 \pm 12^{\# \#}$ & $54 \pm 12^{\#}$ & $60 \pm 16$ & 0.000 \\
\hline $\mathrm{LDL}$ cholesterol $\mathrm{mg} \cdot \mathrm{dL}^{-1}$ & $127 \pm 33$ & $141 \pm 30^{\#}$ & $129 \pm 29$ & $121 \pm 35$ & 0.139 \\
\hline Uric acid $\mathrm{mg} \cdot \mathrm{dL}^{-1}$ & $6.2(4.8-7.1)^{\# \#}$ & $5.3(4.0-6.9)$ & $5.4(4.6-6.7)$ & $4.6(3.9-5.5)$ & 0.035 \\
\hline Haemoglobin $\mathrm{g} \cdot \mathrm{dL}^{-1}$ & $14.8(13.2-15.9)$ & $13.7(13.2-15.3)$ & $14.1(13.6-15.7)$ & $14.3(13.5-14.9)$ & 0.759 \\
\hline Haematocrit \% & $44.2(39.1-46.9)$ & $41.6(38.9-46.4)$ & $41.4(39.3-45.1)$ & $41.2(39.3-43.4)$ & 0.593 \\
\hline Leukocytes $10^{3} \cdot \mathrm{uL}^{-1}$ & $6.944 \pm 1.926$ & $6.898 \pm 2.064$ & $6.800 \pm 1.634$ & $6.862 \pm 2.266$ & 0.983 \\
\hline Platelets $10^{3} \cdot \mathrm{uL}^{-1}$ & $244 \pm 61$ & $255 \pm 72$ & $251 \pm 60$ & $258 \pm 91$ & 0.708 \\
\hline $\mathrm{D}$-dimer $\mathrm{ng} \cdot \mathrm{mL}^{-1}$ & $355(230-615)^{\# \#, 9}$ & $345(238-520)$ & 335 (215-498) & $300(200-380)$ & 0.039 \\
\hline Soluble P-selectin $\mathrm{ng} \cdot \mathrm{mL}^{-1}$ & $2.1 \pm 0.5$ & $2.0 \pm 0.4$ & $2.2 \pm 0.9$ & $1.9 \pm 1.2$ & 0.390 \\
\hline PAI-1 $\mathrm{ng} \cdot \mathrm{mL}^{-1}$ & $4.2(2.5-6.5)^{\#,+}$ & $3.6(2.0-6.1)$ & $2.2(1.2-5.0)$ & $1.5(1.1-5.1)$ & 0.053 \\
\hline $\mathrm{F} 1+2 \mathrm{pmol} \cdot \mathrm{L}^{-1}$ & $303(197-435)^{\# \#,+}$ & $143(101-217)$ & $193(150-217)$ & $162(141-196)$ & 0.002 \\
\hline TAT $\mu g \cdot L^{-1}$ & $4.5(3.2-12.1)^{\#}$ & $3.0(1.9-11.6)^{\#}$ & $2.2(1.0-10.2)^{\pi}$ & $7.9(6.2-10.1)$ & 0.097 \\
\hline
\end{tabular}

Data are presented as $\mathrm{n}(\%)$, mean $\pm \mathrm{SD}$, median (interquartile range) or $\%$, unless otherwise stated. PE: pulmonary embolism; CT90\%: percentage of total study time spent with arterial oxygen saturation $\left(\mathrm{SaO}_{2}\right)<90 \%$; HDL: high-density lipoprotein; LDL: low-density lipoprotein; PAI-1:

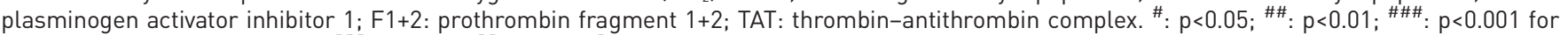

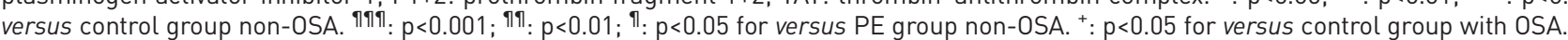




\section{Haematological and biochemical analysis}

The number of circulating erythrocytes, leucocytes and platelets were similar between groups. Dyslipidaemia was significantly more frequent in the PE group than in controls, with both higher triglycerides and lower high-density lipoprotein (HDL)-C levels. C-OSA had lower HDL-C values compared with C non-OSA. Furthermore, uric acid levels were also higher in PE-OSA than C non-OSA subjects $(\mathrm{p}<0.05)($ table 1$)$.

\section{Procoagulant, platelet activity and fibrinolysis biomarkers}

The primary outcome of our study was to compare the percentage of patients with elevated D-dimer between the study groups. The prevalence of elevated levels of D-dimer $\left(>500 \mathrm{ng} \cdot \mathrm{mL}^{-1}\right)$ after stopping anticoagulant treatment for PE was higher in patients with OSA than in patients without OSA (35.4\% versus $19 \%, \mathrm{p}=0.003)$. In the same way, C-OSA had a higher percentage of subjects with high D-dimer levels than $\mathrm{C}$ non-OSA (figure 2). PE patients with OSA had significantly greater D-dimer plasma concentrations than non-OSA (with or without previous PE, $355 \mathrm{ng} \cdot \mathrm{mL}^{-1}\left(230-615 \mathrm{ng} \cdot \mathrm{mL}^{-1}\right)$ versus $345 \mathrm{ng} \cdot \mathrm{mL}^{-1}$ $\left(238-520 \mathrm{ng} \cdot \mathrm{mL}^{-1}\right)$ and $300 \mathrm{ng} \cdot \mathrm{mL}^{-1}\left(200-380 \mathrm{ng} \cdot \mathrm{mL}^{-1}\right) ; \mathrm{p}<0.01$ and $\mathrm{p}<0.05$, respectively). $\mathrm{F} 1+2$ and PAI-1 levels were also higher in patients with PE and OSA compared with those who did not have a PE (with or without OSA) (table 1). Furthermore, TAT was also higher in PE than controls without PE (table 1).

In patients with $\mathrm{PE}, \mathrm{AHI}$ was related to high $\mathrm{D}$-dimer levels (table 2). This association remained significant after adjusting for sex, age, BMI, neck diameter, physical activity level, classic risk factors for pulmonary embolism, blood pressure values and FEV1 \% pred, adjusted OR 1.114 (95\% CI 1019-1218, p=0.018).

The relationships between plasma levels of haemostasis/fibrinolysis biomarkers and sleep parameters, adjusted for the classic risk factors for PE, are shown in table 3. In all study subjects, D-dimer levels were directly related to AHI $(\mathrm{r}=0.279, \mathrm{p}=0.007)$, percentage of total time study spent with $\mathrm{SaO}_{2}<90 \%$ and desaturation index and inversely related to the mean and minimum night-time $\mathrm{SaO}_{2}$ (figure 3). A significant relationship was also found between $\mathrm{AHI}$ and the sP-selectin level. In addition, other significant relationships were found between some indices of nocturnal oxygen saturation and PAI-1 and F1+2 levels (table 3). In a multiple linear regression analysis, adjusted for sex, age, BMI and classic risk factors for pulmonary embolism, we found that $\mathrm{D}$-dimer levels were mainly determined by AHI $(\beta=0.22, p=0.02$, $\left.\Delta \mathrm{R}^{2}=0.12\right)$ and minimum night-time $\mathrm{SaO}_{2}\left(\beta=-0.25, \mathrm{p}=0.011\right.$, and $\left.\Delta \mathrm{R}^{2}=0.04\right)$, whereas the mean sleep $\mathrm{SaO}_{2}$, Epworth Sleepiness Scale and the percentage of total study time spent with $\mathrm{SaO}_{2}<90 \%$ were the main determinants of $\mathrm{F} 1+2$ levels (table 4 ).

Finally, we evaluated the relationship between procoagulant, platelet activity and fibrinolysis biomarkers and sleep characteristics in the subgroup of subjects with OSA and excessive sleepiness (AHI $>5 \mathrm{~h}^{-1}$ and ESS >11). After adjusting for classic risk factors for PE, D-dimer remained significantly related with AHI $(\mathrm{r}=0.631, \mathrm{p}=0.037)$ (figure S1), desaturation index $(\mathrm{r}=0.631, \mathrm{p}=0.021)$ and mean and minimum night-time $\mathrm{SaO}_{2}(\mathrm{r}=-0.515, \mathrm{p}=0.049 ; \mathrm{r}=-0.534, \mathrm{p}=0.049$, respectively). In these patients, AHI was also related to sP-selectin levels $(\mathrm{r}=0.658, \mathrm{p}=0.020$ ) (figure $\mathrm{S} 1$ ), whereas ESS was significantly correlated with PAI-1 $(\mathrm{r}=0.651, \mathrm{p}=0.041), \mathrm{F} 1+2(\mathrm{r}=0.746, \mathrm{p}=0.008)$ and TAT $(\mathrm{r}=0.617, \mathrm{p}=0.033)$.

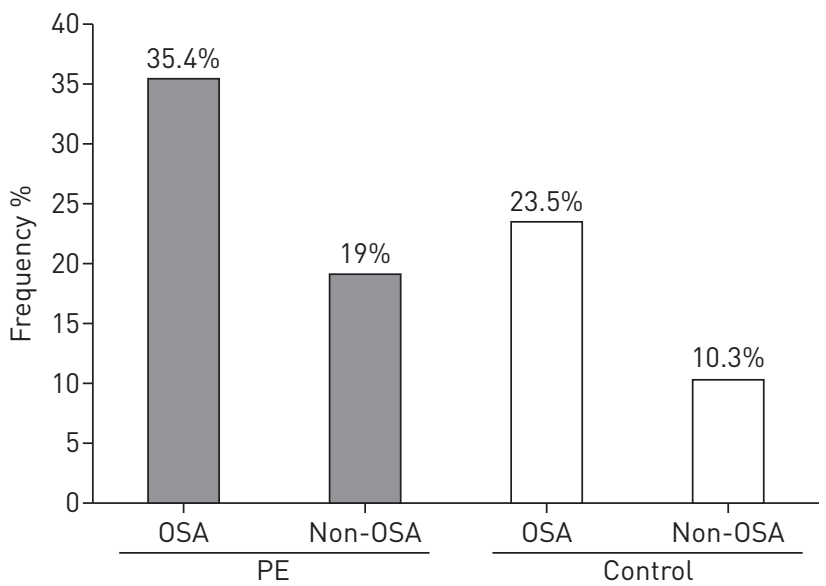

FIGURE 2 Frequencies of high plasmatic levels of D-dimer $\left(>500 \mathrm{ng} \cdot \mathrm{mL}^{-1}\right)$ in patients with pulmonary embolism after anticoagulation discontinuation and in control subjects. $p=0.003$. 
TABLE 2 Risk factors related with the persistence of a high D-dimer plasmatic level after anticoagulant treatment in patients with pulmonary embolism

\begin{tabular}{llc} 
& Crude OR (95\% CI) & p-value \\
\hline Sex male & $0.799(0.318-2.008)$ & 0.633 \\
Age years & $1.030(0.995-1.067)$ & 0.095 \\
Body mass index & $1.024(0.934-1.123)$ & 0.611 \\
Neck diameter & $1.036(0.920-1.167)$ & 0.558 \\
Physical activity & & 0.248 \\
$\quad$ Moderate & $0.778(0.176-3.431)$ & 0.740 \\
$\quad$ High & $0.263(0.047-1.476)$ & 0.129 \\
Forced expiratory volume at 1 s \% pred & $1.027(0.995-1.060)$ & 0.103 \\
Blood pressure & & \\
$\quad$ Systolic & $1.006(0.979-1.034)$ & 0.653 \\
$\quad$ Diastolic & $1.009(0.964-1.057)$ & 0.692 \\
Apnoea-hypopnoea index & $1.030(1.004-1.057)$ & 0.024 \\
\hline
\end{tabular}

OR: odds ratio; $95 \% \mathrm{Cl}$ : 95\% confidence interval.

\section{Discussion}

In this study, we have shown that PE patients with OSA had higher rates of elevated D-dimer levels $\left(>500 \mathrm{ng} \cdot \mathrm{mL}^{-1}\right.$ ) after anticoagulation discontinuation for PE than in patients without OSA. Furthermore, D-dimer levels were higher in OSA patients than in patients without OSA. D-dimer concentrations were directly related to the severity of OSA, and inversely correlated with nocturnal hypoxia.

There is increasing evidence from cross-sectional and longitudinal studies, that OSA is an independent risk factor for PE [1-5]. We have recently shown in a group of $107 \mathrm{PE}$ patients significantly higher prevalence in all OSA severity groups when compared with controls.

We found significant association between $\mathrm{AHI}$ and PE after adjustment for the main confounding factors. The adjusted OR using AHI as a continuous variable was 1.038, which indicated that for every 10-unit rise in AHI, PE risk increased by $45 \%$ [1].

The mechanisms explaining this novel association between PE and OSA are unclear. PE is the result of Virchow's classic risk triad, namely vascular endothelial impairment, stasis of blood flow, and/or increased coagulability [16]. OSA could potentially play a role in all these three mechanistic pathways. Apnoea increase oxidative stress [17], and activate inflammatory cascade that impairs endothelial function [18].

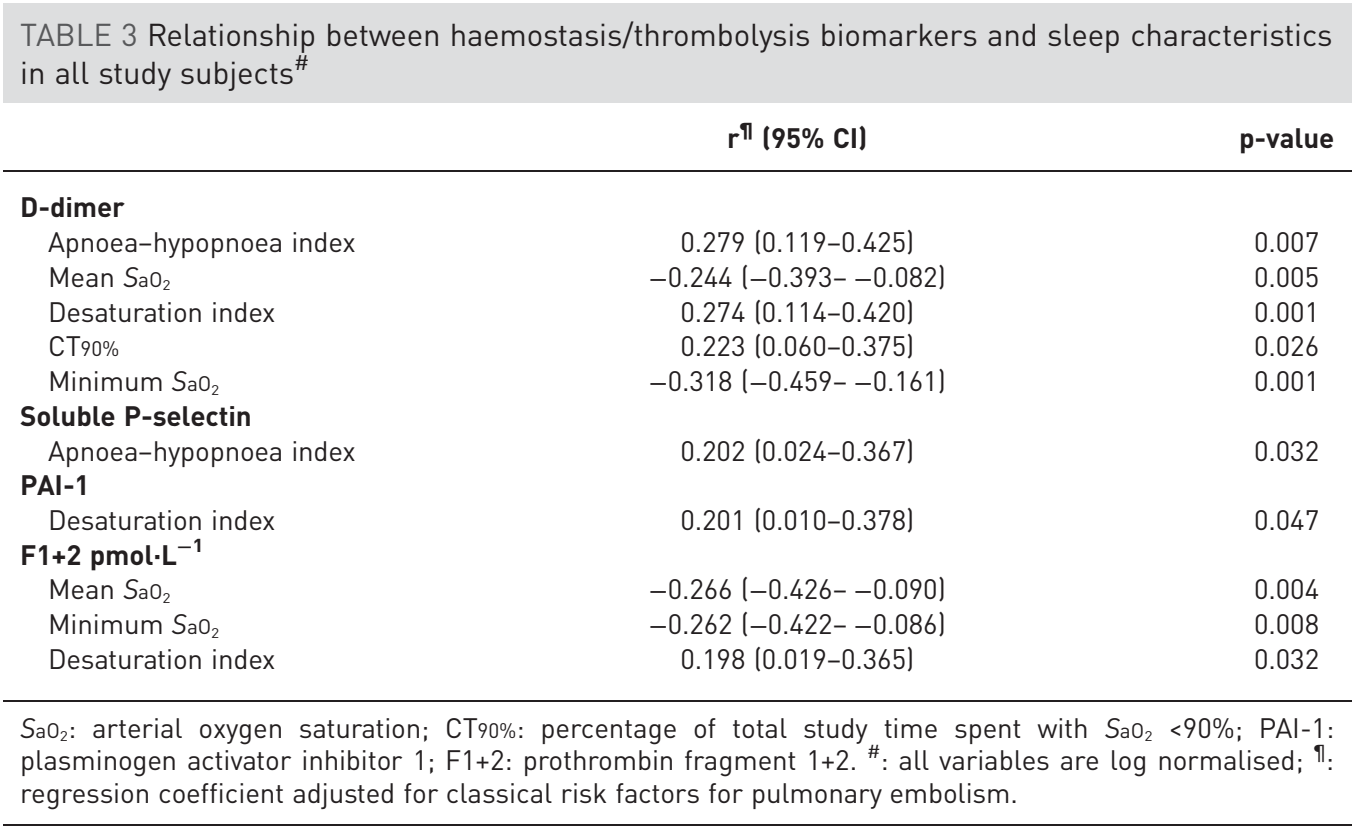



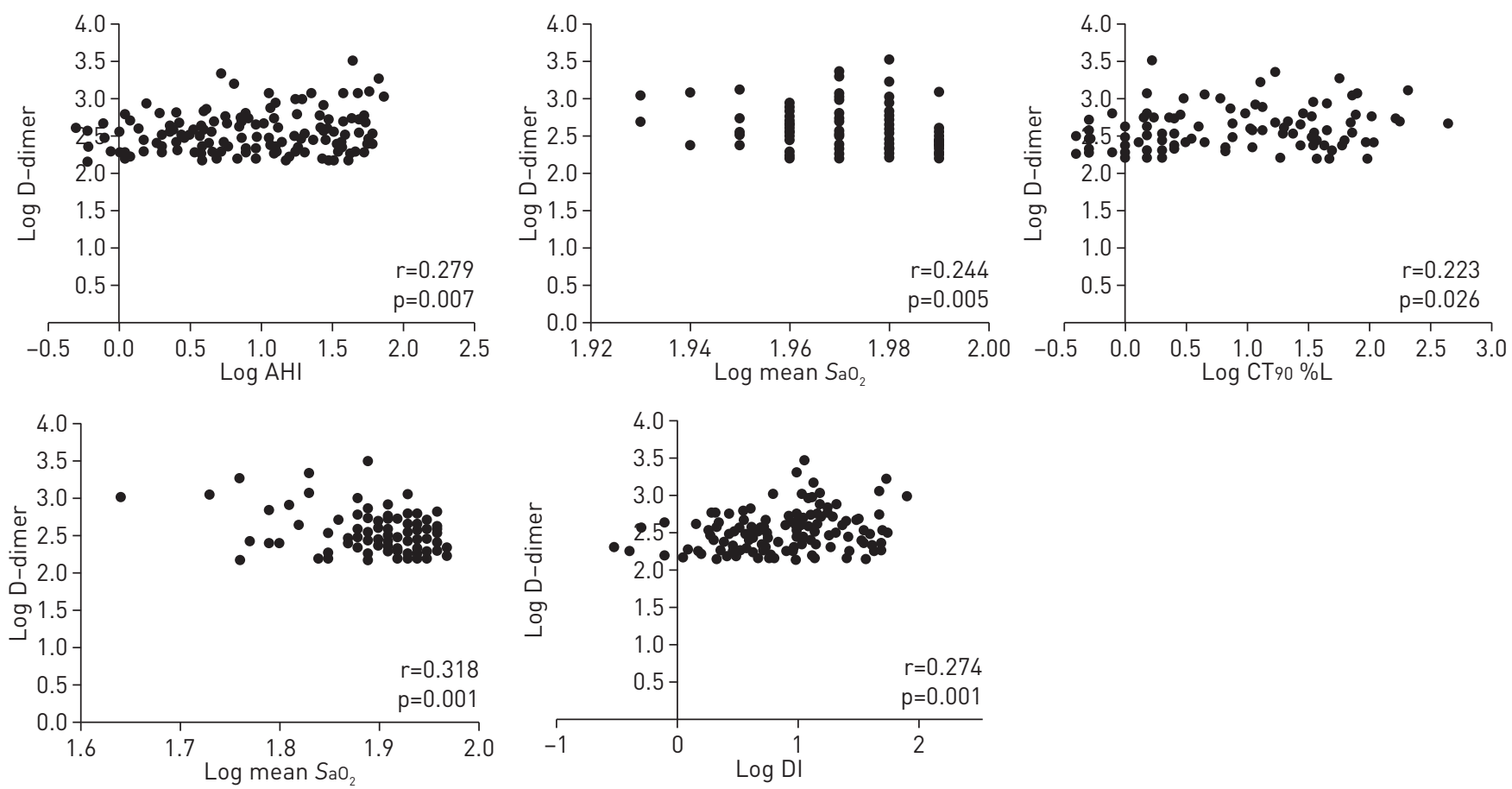

FIGURE 3 Relationship between the sleep parameters and levels of D-dimer in the whole study subjects. Regression coefficients are adjusted for classic risk factors for pulmonary embolism and all variables are log normalised. AHI: Apnoea-hypopnoea index; $\mathrm{SaO}_{2}$ : arterial oxygen saturation; CT90\%: the percentage of total study time spent with $\mathrm{SaO}_{2}<90 \%$; DI: desaturation index.

OSA-related haemodynamic alterations may slow intravenous flow [19], and lastly, cross-sectional studies support increased coagulability, platelet activity and aggregability $[20,21]$ and decreased fibrinolytic capacity in OSA. It has also been shown that that coagulant activity, platelet function and fibrinolytic system are improved after continuous positive airway pressure (CPAP) [20-25]. However, the associations with OSA and changes with CPAP have not been uniformly established for all biomarkers [24-26].

D-dimer is a major plasmin-derived degradation product of cross-linked fibrin. Its generation depends on the presence of fibrin and plasmin. The level of D-dimer is considered to reflect the global activity of clot formation and lysis [10].

SHITRIT et al. [27] selected 103 OSA patients and found high D-dimer levels in 14. The subgroup with high D-dimer levels had lower minimal oxygen saturation and a longer mean period with $\mathrm{SaO}_{2}<90 \%$ in the sleep study compared with normal D-dimer OSA group [27]. In keeping with these results, it has been shown that D-dimer increased significantly when patients were exposed to hypoxic conditions [28]. Other investigators have found augmented fibrinogen levels in both adults and children with OSA [29-31],

TABLE 4 Independent determinants of D-dimer and prothrombin fragment $1+2(F 1+2)$ levels ${ }^{\#}$

Unstandardised regression coefficients $\mathrm{B} \pm \mathrm{sE}(95 \% \mathrm{CI})$

\section{Standardised regression coefficients $B$}

\begin{tabular}{|c|c|c|c|c|c|}
\hline \multicolumn{6}{|l|}{$\mathrm{D}$-dimer $\mathrm{mg} \cdot \mathrm{mL}^{-1}$} \\
\hline Constant & $1.325 \pm 0.396(0.540-2.110)$ & & 0.001 & & \\
\hline Minimum $\mathrm{SaO}_{2} \%$ & $-0.0121 \pm 0.005(-0.021--0.003)$ & -0.246 & 0.011 & 0.120 & 0.120 \\
\hline $\mathrm{AHI}$ events $\cdot \mathrm{h}^{-1}$ & $0.005463 \pm 0.002(0.00-0.010)$ & 0.224 & 0.020 & 0.160 & 0.040 \\
\hline Constant & $7414.0 \pm 2214$ (3015-11813) & & 0.001 & & \\
\hline Mean $\mathrm{SaO}_{2} \%$ & $-77.2 \pm 23.2(-123.3--31.1)$ & -0.427 & 0.001 & 0.092 & 0.092 \\
\hline ESS & $29.9 \pm 11.9(6.4-53.5)$ & 0.251 & 0.013 & 0.144 & 0.052 \\
\hline
\end{tabular}

$\mathrm{SaO}_{2}$ : arterial oxygen saturation; $\mathrm{AHI}$ : apnoea-hypopnoea index; ESS: Epworth Sleepiness Scale; CT90\%: the percentage of total study time spent with $\mathrm{SaO}_{2}<90 \%$. " : values are adjusted for sex, age, body mass index and classic risk factors for pulmonary embolism. 
which decreased after CPAP treatment [22]. However, these findings are not uniform with several studies showing no, or even inverse, associations between OSA and D-dimer [30, 32, 33], while a number of reports did not find changes after CPAP [24, 32-34]. We also found significantly higher D-dimer plasma levels in PE-OSA compared with non-OSA PE patients. Furthermore D-dimer was directly related to OSA severity and inversely correlated to nocturnal hypoxaemia. Nocturnal hypoxia plays an important role in the procoagulant state associated with OSA [23, 25, 27, 28, 31]. Similarly we have shown significant correlations between D-dimer, sP-selectin, PAI-1, F1+2 and TAT and some indices of nocturnal hypoxaemia. Venous thrombosis usually initiates at the venous valves in the legs, where stasis and hypoxia may occur, which can induce endothelial damage starting a potentially hypercoagulable microenvironment $[35,36]$. OSA is associated to sedentary lifestyle and obesity that may induce a procoagulant state and venous stasis, which have been associated with an increased risk of PE [7]. Moreover, it is tempting to speculate that nocturnal hypoxaemia in OSA patients may lead to up-regulation of procoagulant activity in valvular sinus in deep veins.

The conflicting results in D-dimer in OSA may be explained by differences in populations and sampling (concomitant comorbidities, sex distribution, age, BMI and varying degrees of sleep apnoea severity). Besides, non-dipping blood pressure profile at night and emotional and mood factors are associated with elevated D-dimer $[37,38]$, and the impact of these aspects has not been systematically evaluated in the majority of the studies.

The main finding of our study (higher proportion of subjects with elevated post-anticoagulation D-dimer in PE-OSA compared with PE non-OSA) may have important clinical implications. In patients with a first $\mathrm{PE}$ event, an elevated D-dimer level after anticoagulation is stopped is a risk factor for recurrent PE [10]. A randomised clinical trial has shown that patients with an abnormal D-dimer test and who restarted anticoagulation had a significantly lower combined incidence of recurrent venous thromboembolism and bleeding, than those who did not resume the treatment [11]. Therefore, it has been suggested to use this biomarker as a tool to choose the extent of anticoagulation. We hypothesise that those patients with $\mathrm{PE}$ and concomitant OSA have an increased risk of future PE episodes, and it could be used to identify patients with a high risk of recurrence and who might require longer anticoagulant treatment.

Other global coagulation markers activation and fibrinolysis, such as TAT, sP-selectin, PAI-1 and F1+2, have been explored as predictors of recurrent venous thromboembolism, but are not yet ready for clinical use [12-15].

Several studies have shown increased sP-selectin, PAI-1 and TAT levels in OSA [23, 25, 26, 28, 30, 32]. However, there are conflicting results $[24,26]$. But, overall, most studies have confirmed that OSA is associated with a procoagulant state.

We could not demonstrate any difference in sP-selectin, F1+2, PAI-1, and TAT between OSA and non-OSA subjects. Consequently, we found no imbalance in platelet activation, fibrinolysis system and thrombin generation. However, there were significant correlations between sP-selectin, PAI-1, F1+2 and TAT and some indices of nocturnal hypoxaemia. These could be explained because they are really unrelated, or because the present study might have had limited power to detect differences. The precise processes that initiate venous thrombosis remain uncertain. Several studies have shown increased markers of coagulation activation and platelet activity such as D-dimer, TAT and $\mathrm{F} 1+2$, and sP-selectin in conditions with enhanced thrombin formation [39]. A single abnormality factor is seldom enough to cause venous thrombosis, as it may require a synergy of several factors. ToukH et al. [40] used thromboelastography to assess coagulability, which provides information about the full spectrum of the haemostatic process, (from the initial formation of fibrin until lysis of the clot) and found that OSA patients had a procoagulant state that was reduced after 2 weeks of CPAP. As a result, there may be a thrombosis threshold where the trend to cause thrombin is not effectively regulated by antithrombotic mechanisms.

There are a number of limitations that should be considered in relation to our study, beyond sample size, which limits some ancillary subanalyses. First, there is a marked diurnal variability in procoagulant factors in OSA [32, 33, 41]. We did single time-point assessments, but whole samples in all subjects were collected after fasting overnight between 08:00 and 10:00 h, and single sample assessments of elevated D-dimer have been prospectively related with an increased risk of recurrent PE in several studies [10]. Second, although we designed our study to evaluate coagulation markers in addition to our primary outcome of association between OSA and PE, the selection of these biomarkers was determined post hoc, thus the study may have been underpowered to find out changes in some hypercoagulable state mediators. Third, although cross-sectionally high D-dimer concentration in OSA patients compared with non-OSA subjects is consistent with a procoagulant state, any cause-effect relationship would require confirmation in prospective longitudinal cohort studies. Fourth, although OSA diagnosis was based on type 3 portable sleep monitoring system without electroencephalographic signals, it has been previously validated. Both 
patients and controls were studied with the same device and protocol, which could underestimate the severity of OSA and the relationships that we have found between D-dimer level and OSA severity. Finally, we acknowledge our patients have comorbidities that could affect the values of some of the biomarkers tested. However, the groups were quite similar and we selected consecutive PE patients that correspond to the real-life entire clinical disease spectrum of PE.

To conclude, we have found that PE patients with associated OSA had a higher proportion of subjects with high post-anticoagulation D-dimer, than in those without OSA. Moreover, D-dimer levels were higher in PE patients with associated OSA than without, indicating that there is a persistent hypercoagulable state in OSA. Data from new studies are needed to definitively clarify if OSA is a risk factor for PE recurrence, particularly in those ones with high D-dimer values. Given the high prevalence of OSA in PE patients [1-5], the increased blood coagulability induced by OSA, and the raised percentage of patients with elevated D-dimer levels after the completion of anticoagulation, the potential of CPAP and/or resumption anticoagulant drugs to reduce incidence and mortality in patients with OSA clearly warrants further study, in particular for patients with previous PE.

\section{Acknowledgements}

We thank Margalida Bosch (Dept of Pneumology, University Hospital Son Espases, Palma de Mallorca, Spain), Mónica Iglesias, Ángel Ríos and Meritxell López (all IdISPa- FISIB, Hospital Universitari Son Espases, Palma de Mallorca, Spain) for their assistance in the fieldwork of the study.

\section{References}

1 Alonso-Fernández A, de la Peña M, Romero D, et al. Association between obstructive sleep apnea and pulmonary embolism. Mayo Clin Proc 2013; 88: 579-587.

2 Ambrosetti M, Lucioni A, Ageno W, et al. Is venous thromboembolism more frequent in patients with obstructive sleep apnea syndrome? J Thromb Haemost 2004; 2: 1858-1860.

3 Arnulf I, Merino-Andreu M, Perrier A, et al. Obstructive sleep apnea and venous thromboembolism. JAMA 2002; 287: 2655-2656.

4 Arzt M, Luigart R, Schum C, et al. Sleep-disordered breathing in deep vein thrombosis and acute pulmonary embolism. Eur Respir J 2012; 40: 919-924.

5 Chou KT, Huang CC, Chen YM, et al. Sleep apnea and risk of deep vein thrombosis: a non-randomized, pair-matched cohort study. Am J Med 2012; 125: 374-380.

6 Durán J, Esnaola S, Rubio R, et al. Obstructive sleep apnea-hypopnea and related clinical features in a population-based sample of subjects aged 30 to 70 yr. Am J Respir Crit Care Med 2001; 163: 685-689.

7 Torbicki A, Perrier A, Konstantinides S, et al. Guidelines on the diagnosis and management of acute pulmonary embolism: the Task Force for the Diagnosis and Management of Acute Pulmonary Embolism of the European Society of Cardiology (ESC). Eur Heart J 2008; 29: 2276-2315.

8 Marin JM, Carrizo SJ, Vicente E, et al. Long-term cardiovascular outcomes in men with obstructive sleep apnoea-hypopnoea with or without treatment with continuous positive airway pressure: an observational study. Lancet 2005; 365: 1046-1053.

9 Heit JA, Mohr DN, Silverstein MD, et al. Predictors of recurrence after deep vein thrombosis and pulmonary embolism: a population-based cohort study. Arch Intern Med 2000; 160: 761-768.

10 Eichinger S, Minar E, Bialonczyk C, et al. D-dimer levels and risk of recurrent venous thromboembolism. JAMA 2003; 290: 1071-1074.

11 Palareti G, Cosmi B, Legnani C, et al. D-dimer testing to determine the duration of anticoagulation therapy. N Engl J Med 2006; 355: 1780-1789.

12 de Raucourt E, Meyer G, Landais P, et al. Markers of hemostatic system activation in pulmonary embolism: changes during and after cessation of anticoagulant treatment. Blood Coagul Fibrinolysis 2000; 11: 249-253.

13 Kyrle PA, Hron G, Eichinger S, et al. Circulating P-selectin and the risk of recurrent venous thromboembolism. Thromb Haemost 2007; 97: 880-883.

14 Poli D, Antonucci E, Cecchi E, et al. Clotting activation after oral anticoagulant therapy discontinuation: a risk factor for recurrent venous thromboembolism. Blood Coagul Fibrinolysis 2004; 15: 221-225.

15 Kyrle PA, Eichinger S, Pabinger I, et al. Prothrombin fragment F1+2 is not predictive for recurrent venous thromboembolism. Thromb Haemost 1997; 77: 829-833.

16 Rosendaal FR. Venous thrombosis: the role of genes, environment, and behavior. Hematology Am Soc Hematol Educ Program 2005; 2005: 1-12.

17 Alonso-Fernández A, García-Río F, Arias MA, et al. Effects of CPAP on oxidative stress and nitrate efficiency in sleep apnoea: a randomised trial. Thorax 2009; 64: 581-586.

18 Arias MA, García-Río F, Alonso-Fernández A, et al. CPAP decreases plasma levels of soluble tumour necrosis factor-alpha receptor 1 in obstructive sleep apnoea. Eur Respir J 2008; 32: 1009-1015.

19 Kasai T, Floras JS, Bradley TD. Sleep apnea and cardiovascular disease: a bidirectional relationship. Circulation 2012; 126: 1495-1510.

20 Geiser T, Buck F, Meyer BJ, et al. In vivo platelet activation is increased during sleep in patients with obstructive sleep apnea syndrome. Respiration 2002; 69: 229-234.

21 Hui DS, Ko FW, Fok JP, et al. The effects of nasal continuous positive airway pressure on platelet activation in obstructive sleep apnea syndrome. Chest 2004; 125: 1768-1775.

22 Chin K, Ohi M, Kita H, et al. Effects of NCPAP therapy on fibrinogen levels in obstructive sleep apnea syndrome. Am J Respir Crit Care Med 1996; 153: 1972-1976.

23 Minoguchi K, Yokoe T, Tazaki T, et al. Silent brain infarction and platelet activation in obstructive sleep apnea. Am J Respir Crit Care Med 2007; 175: 612-617. 
Phillips CL, McEwen BJ, Morel-Kopp MC, et al. Effects of continuous positive airway pressure on coagulability in obstructive sleep apnoea: a randomised, placebo-controlled crossover study. Thorax 2012; 67: 639-644.

von Känel R, Loredo JS, Ancoli-Israel S, et al. Association between sleep apnea severity and blood coagulability: treatment effects of nasal continuous positive airway pressure. Sleep Breath 2006; 10: 139-146.

26 Robinson GV, Pepperell JC, Segal HC, et al. Circulating cardiovascular risk factors in obstructive sleep apnoea: data from randomised controlled trials. Thorax 2004; 59: 777-782.

27 Shitrit D, Peled N, Shitrit AB, et al. An association between oxygen desaturation and D-dimer in patients with obstructive sleep apnea syndrome. Thromb Haemost 2005; 94: 544-547.

28 von Känel R, Loredo JS, Powell FL, et al. Short-term isocapnic hypoxia and coagulation activation in patients with sleep apnea. Clin Hemorheol Microcirc 2005; 33: 369-377.

29 Kaditis AG, Alexopoulos EI, Kalampouka E, et al. Morning levels of fibrinogen in children with sleep-disordered breathing. Eur Respir J 2004; 24: 790-797.

30 Mehra R, Xu F, Babineau DC, et al. Sleep disordered breathing and pro-thrombotic biomarkers: cross-sectional results of the Cleveland Family Study. Am J Respir Crit Care Med 2010; 182: 826-833.

31 Wessendorf TE, Thilmann AF, Wang YM, et al. Fibrinogen levels and obstructive sleep apnea in ischemic stroke. Am J Respir Crit Care Med 2000; 162: 2039-2042.

32 von Känel R, Natarajan L, Ancoli-Israel S, et al. Day/night rhythm of hemostatic factors in obstructive sleep apnea. Sleep 2010; 33: 371-377.

33 von Känel R, Natarajan L, Ancoli-Israel S, et al. Effect of continuous positive airway pressure on day/night rhythm of prothrombotic markers in obstructive sleep apnea. Sleep Med 2013; 14: 58-65.

34 McEwen BJ, Phillips CL, Morel-Kopp MC, et al. Diurnal changes and levels of fibrin generation are not altered by continuous positive airway pressure (CPAP) in obstructive sleep apnoea (OSA): a randomised, placebo-controlled crossover study. Thromb Haemost 2012; 108: 701-709.

35 Shreeniwas R, Ogawa S, Cozzolino F, et al. Macrovascular and microvascular endothelium during long-term hypoxia: alterations in cell growth, monolayer permeability, and cell surface coagulant properties. J Cell Physiol 1991; 146: 8-17.

36 Hamer JD, Malone PC, Silver IA. The $\mathrm{PO}_{2}$ in venous valve pockets: its possible bearing on thrombogenesis. $\mathrm{Br} J$ Surg 1981; 68: 166-170.

37 von Känel R, Bardwell WA, Berry CC, et al. Independent contribution of psychological factors to fibrin turnover in subjects with sleep apnoea and/or systemic hypertension. Clin Sci (Lond) 2002; 103: 331-337.

38 von Känel R, Jain S, Mills PJ, et al. Relation of nocturnal blood pressure dipping to cellular adhesion, inflammation and hemostasis. J Hypertens 2004; 22: 2087-2093.

39 Pabinger I, Ay C. Biomarkers and venous thromboembolism. Arterioscler Thromb Vasc Biol 2009; 29: 332-336.

40 Toukh M, Pereira EJ, Falcon BJ, et al. CPAP reduces hypercoagulability, as assessed by thromboelastography, in severe obstructive sleep apnoea. Respir Physiol Neurobiol 2012; 183: 218-223.

41 Barceló A, Piérola J, de la Peña M, et al. Impaired circadian variation of platelet activity in patients with sleep apnea. Sleep Breath 2012; 16: 355-360. 